\title{
Repetitive Colonoscopic Decompression as a Bridge Therapy before Surgery in a Pregnant Patient with Chronic Intestinal Pseudo-Obstruction
}

\author{
Joon Sung Kim¹, Bo-In Lee', Byung-Wook Kim¹, Hwang Choi', Yun-Seok Lee ${ }^{2}$ and Leeso Maeng ${ }^{3}$ \\ Departments of ${ }^{1}$ Internal Medicine, ${ }^{2}$ General Surgery, and ${ }^{3}$ Pathology, The Catholic University of Korea College of Medicine, Seoul, Korea
}

\begin{abstract}
Chronic intestinal pseudo-obstruction is a rare clinical syndrome which is characterized by intestinal obstruction without occluding lesions in the intestinal lumen and pregnancy is one of the important aggravating factors. Here, we report a case of a woman with intractable intestinal pseudo-obstruction that was precipitated by pregnancy. She could not make any stool passage for more than 4 weeks until a fetal gestational age of 17 weeks was reached. However, the patient could be maintained by repetitive colonoscopic decompressions and finally total colectomy could be performed successfully at a fetal gestational age of 21 weeks.
\end{abstract}

Key Words: Chronic intestinal pseudo-obstruction; Pregnancy; Colonoscopic decompression; Interstitial cells of Cajal

\section{INTRODUCTION}

Intestinal pseudo-obstruction is a clinical syndrome that involves bowel obstruction without any mechanical cause. ${ }^{1}$ It can be either acute or chronic and may occur in the small bowel or the colon. Chronic intestinal pseudo-obstruction (CIP) usually exhibits a relapsing clinical course and may either be idiopathic or occur due to other systemic disorders. Pregnancy can be a precipitating factor of colonic pseudo-obstruction. ${ }^{2}$ Recently, we experienced a case of a woman with intractable colonic pseudo-obstruction that was aggravated after pregnancy. Endoscopic decompression could delay surgical treatment until a gestational age of 21 weeks and finally a full-term delivery could be achieved.

Received: September 4, 2012 Revised: December 6, 2012

Accepted: February 12, 2013

Correspondence: Bo-In Lee

Division of Gastroenterology, Department of Internal Medicine, Incheon St. Mary's Hospital, The Catholic University of Korea College of Medicine, 56 Dongsu-ro, Bupyeong-gu, Incheon 403-720, Korea

Tel: +82-32-280-5051, Fax: +82-32-280-5082, E-mail: gidoc4u@gmail.com

(c) This is an Open Access article distributed under the terms of the Creative Commons Attribution Non-Commercial License (http://creativecommons.org/ licenses/by-nc/3.0) which permits unrestricted non-commercial use, distribution, and reproduction in any medium, provided the original work is properly cited.

\section{CASE REPORT}

A 31-year-old woman presented with abdominal pain, distension, and constipation at a fetal gestational age of 17 weeks. The patient's symptoms aggravated at fetal gestational age of 10 weeks and her last pregnancy was aborted 2 years earlier at another hospital due to severe intestinal obstruction. She had suffered from unexplainable obstructive symptoms 5 years earlier and was diagnosed with CIP because of repetitive obstructive symptoms without any visible obstructive lesion observed on abdominopelvic computed tomography (CT), barium enema, or colonoscopy (Fig. 1). Anorectal manometry was performed at another hospital and the results were normal. She was most recently admitted to our hospital 5 months ago because of recurrent obstructive symptoms. Because the intravenous administration of neostigmine was not effective, colonoscopic decompression was performed at that time. The patient was discharged after her condition improved.

At the time of admission, physical examination revealed the presence of abdominal distension and diffuse tenderness. Laboratory examination revealed a white blood cell count of $8,120 / \mathrm{mm}^{3}$, a hemoglobin level of $9.9 \mathrm{~g} / \mathrm{dL}$, a platelet count of $281,000 / \mathrm{mm}^{3}$, a blood sugar level of $99 \mathrm{mg} / \mathrm{dL}$, a blood urea nitrogen level of $12.2 \mathrm{mg} / \mathrm{dL}$, a creatinine level of $1.1 \mathrm{mg} / \mathrm{dL}$, a sodium level of $139 \mathrm{mEq} / \mathrm{L}$, a potassium level of $3.8 \mathrm{mEq} / \mathrm{L}$, a 
chloride level of $104 \mathrm{mEq} / \mathrm{L}$, a calcium level of $8.7 \mathrm{mg} / \mathrm{dL}$, a phosphorous level of $3.6 \mathrm{mg} / \mathrm{dL}$, and a magnesium level of $1.8 \mathrm{mg} / \mathrm{dL}$. Neither radiologic study nor neostigmine administration could be performed because of the risk to the fetus. Colonoscopy was performed to provide decompression because the patient's pain and distension worsened after 5 days of conservative care. The colonoscope was passed just beyond the splenic flexure, at which point the lumen was found to be obstructed by a large fecal bezoar (Fig. 2). Attempts to break the fecal bezoar with an endoscopic snare (Olympus Disposable Electrosurgical Snare SD-210U-10; Olympus Medical Systems Corp., Tokyo, Japan) met with limited success. After suctioning the retained gas and contents of the proximal colon, a drainage catheter (ENBD-7-LIGUORY, 7 Fr, $250 \mathrm{~cm}$; Cook

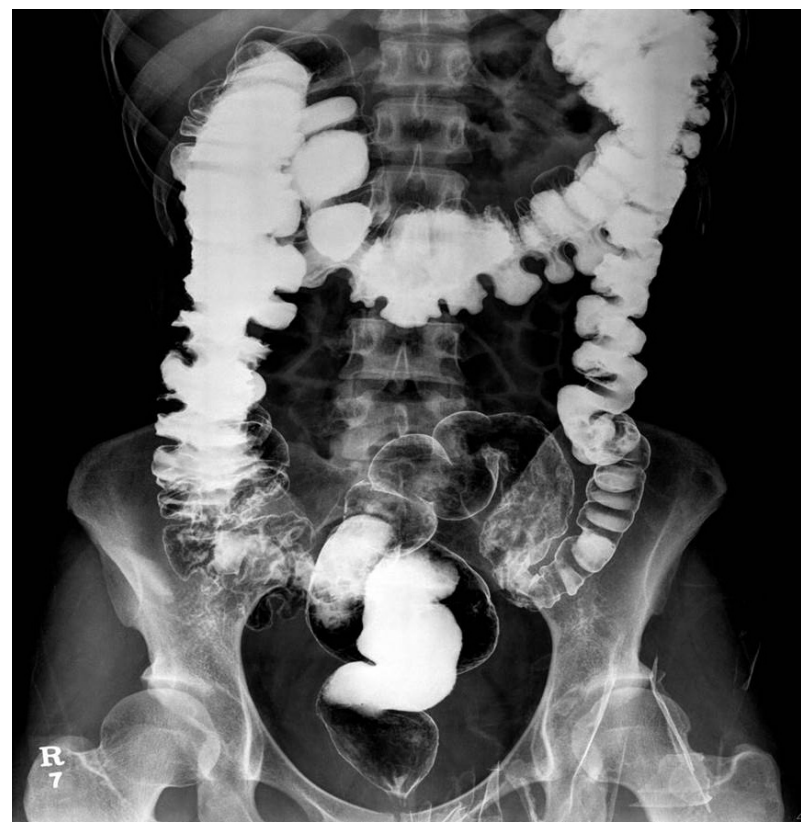

Fig. 1. Barium enema reveals no obstructive lesions.
Medical Inc., Winston-Salem, NC, USA) was placed through the working channel of the endoscope. The catheter was irrigated periodically with normal saline to prevent obstruction and ensure that the intestinal contents and gas were drained effectively. The patient's symptoms improved for a few days after decompression but recurred soon thereafter. Additional two attempts at endoscopic decompression were made and a new drainage catheter was inserted in each of the attempts until surgery could be performed.

Subtotal colectomy with end-ileostomy was performed electively at a fetal gestational age of 21 weeks. Ileorectal anastomosis could not be performed due to the enlarged uterus and was postponed until after delivery. The excised colon measured $100 \mathrm{~cm}$ in length. The proximal portion from the cecum to the descending colon was dilated up to $16 \mathrm{~cm}$ in diameter and a focally narrowed transitional zone was observed around the sigmoid colon (Fig. 3). Full colonic sections were obtained for histopathological analysis, which revealed no apparent histopathological alteration in the muscularis propria or neural plexuses (Fig. 4). However, immunohistochemistry for C-KIT (CD 117) revealed fewer interstitial cells of Cajal (ICCs) in dilated portions of the colon compared to the undilated part (Fig. 5). Stool passage was normalized after surgery and pregnancy was maintained and resulted in a full-term delivery.

\section{DISCUSSION}

Pregnancy was one of the most important precipitating factors contributing to CIP in this case. Elevated progesterone, prostaglandin, and glucagon levels as well as the pressure of the gravid uterus are suggested as factors that exacerbate the pseudo-obstruction experienced by the patient during pregnancy. ${ }^{2}$ Constipation, progressive abdominal pain, and abdominal distension are common symptoms in pregnancy and may delay
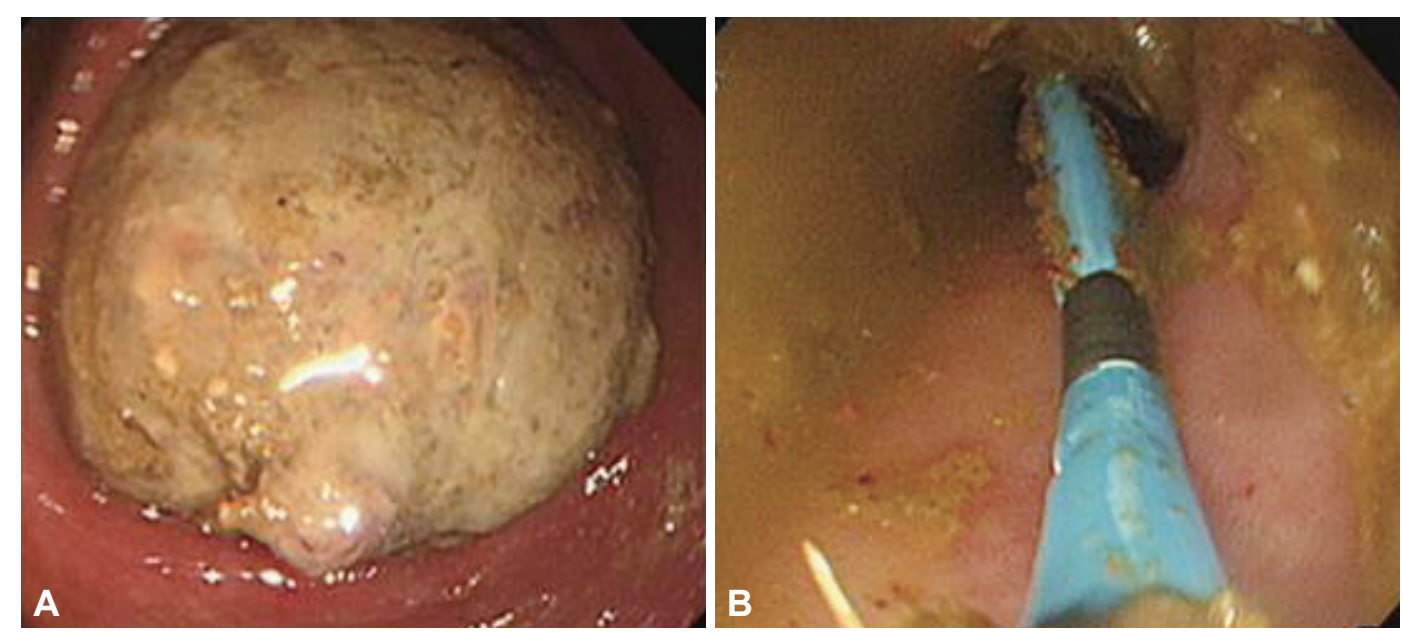

Fig. 2. (A) The fecal bezoar is obstructing the colonic lumen. (B) A decompression rectal tube was inserted beyond the obstruction through the scope. 


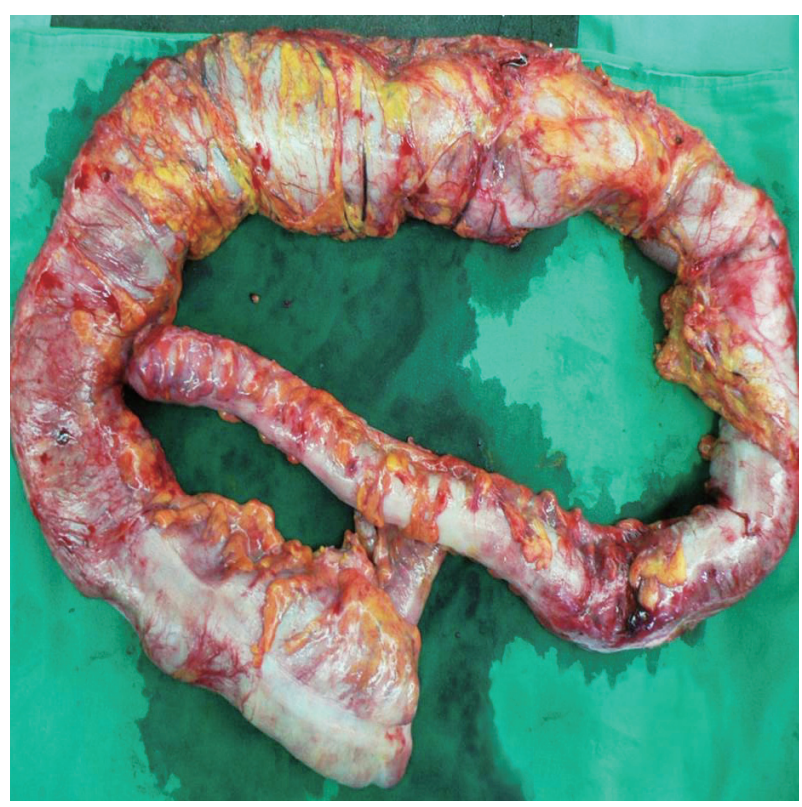

Fig. 3. Gross findings for the resected colon and terminal ileum. The colon measured $100 \mathrm{~cm}$ in length, and the proximal portion was dilated. The diameter of the dilated portion measured $16 \mathrm{~cm}$. No perforation was found. the diagnosis of pseudo-obstruction. Moreover, abdominal radiographs and CT scans, which may be required for evaluation and are typically diagnostic, may be limited in the case of a pregnant woman due to the desire to limit radiation exposure to the fetus. ${ }^{3}$ Fortunately, our patient had already been diagnosed with CIP. Intravenous administration of neostigmine is recommended in patients who do not show improvement after conservative management. Neostigmine may lead to resolution of acute colonic pseudo-obstruction and may also be effective in acute exacerbations of CIP., In this case, the patient's unresponsiveness to neostigmine 5 months earlier and the possible risks to the fetus deterred the authors from using it again.

Endoscopic decompression can be another option for treating colonic pseudo-obstruction when neostigmine is not effective or contraindicated. ${ }^{6}$ Efficacy of endoscopic decompression is increased when a cecal tube is inserted at the time of colonoscopy. Although the cecum could not be intubated due to a large fecal bezoar in our case, placements of a decompression tube beyond the obstruction site was available.

Tubes for colorectal decompression include commercial tubes specifically aimed for decompression, tubes for enteroclysis,
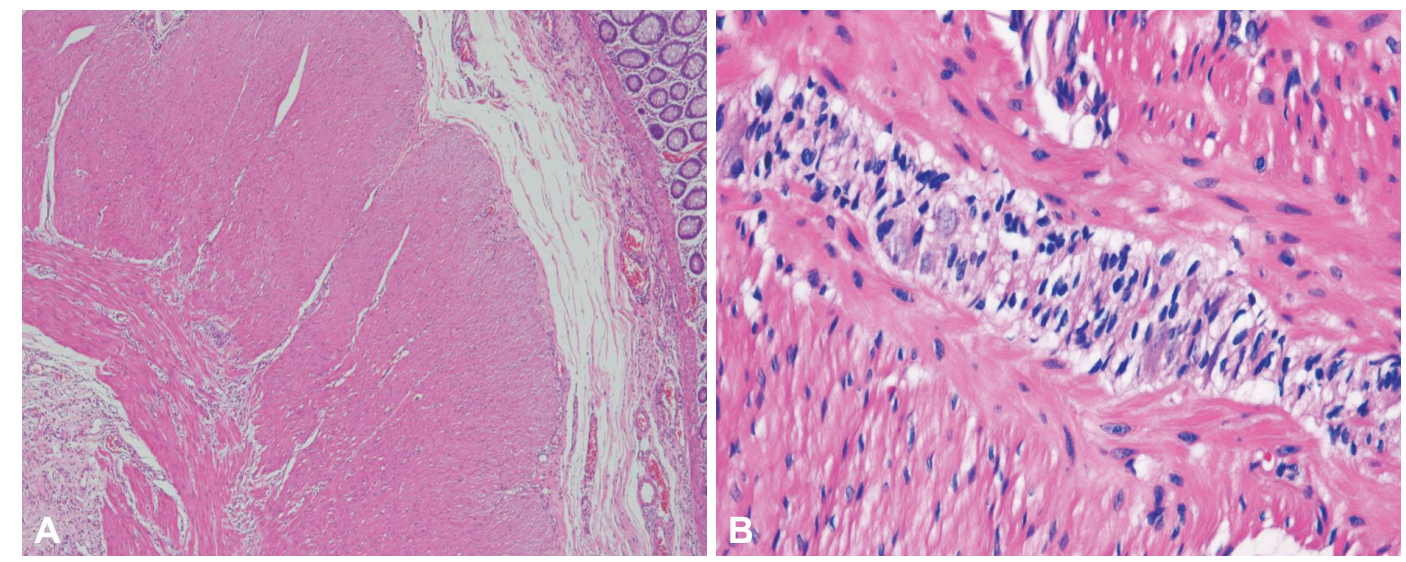

Fig. 4. H\&E stained section of the colon reveals the presence of normal ganglion cells within the myenteric plexus $(A, \times 40 ; B, \times 400)$.
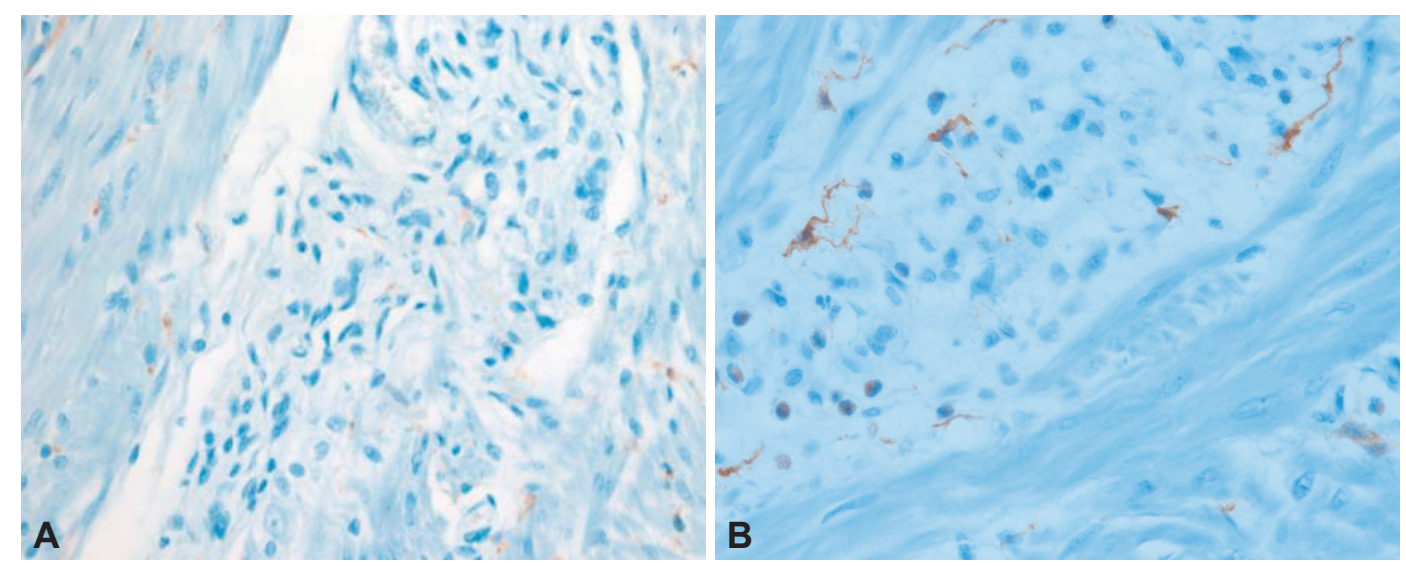

Fig. 5. (A) CD117 immunohistochemical staining shows decreased numbers of interstitial cells of Cajal (ICCs) at the dilated portion of colon ( $\times 400)$. (B) The undilated portion of colon shows good distribution of ICCs $(\times 400)$. 
and nasogastric tubes. However, commercial tubes for colorectal decompression are not currently available in Korea. Moreover, all the tubes above should be inserted by Seldinger technique under fluoroscopic guidance. In contrast, nasobiliary tubes can be easily inserted through the working channel of endoscopy without fluoroscopy. Radiation exposure should be avoided since our patient was pregnant. ${ }^{7}$ Although the small diameter of nasobiliary tubes limits effective decompression, periodic irrigation can prevent plugging of the tube and ensure effective drainage. Colonoscopy may be relatively safe without large fetal risks during the second trimester with limited data during the other trimesters. ${ }^{8}$ Repetitive colonoscopic decompression was able to relieve the patient's symptoms until the second trimester of pregnancy when surgery was available.

CIP can be classified into three major categories based on the underlying pathological abnormality: enteric visceral myopathy, neuropathy, and mesenchymopathy. ${ }^{9}$ This classification is based on the involvement of smooth muscle cells, the enteric nervous system, and ICCs, respectively. CIP can also be classified based on the existence of a transitional zone. Choe et al. ${ }^{10}$ examined the surgical specimens of patients who received surgery for intractable constipation and found that a substantial number of patients presented with a distinct transitional zone with segmental hypoganglionosis. Another study by Do et al. ${ }^{11}$ suggested a novel classification of hypoganglionosis patients into two groups: type I with a focally narrowed transition zone and type II without a transition zone.

Our patient showed dilation of the proximal to mid colon with distinct narrowing around the sigmoid colon. However, pathologic studies of the narrowed segment did not reveal any abnormality in the ganglion cells or the neural plexuses. The only pathologic abnormality were marked reduction of ICCs in multifocal areas of the dilated colon. It has been speculated that alterations in the ICC network may result in impaired control of peristalsis resulting in CIP. However, the diagnostic criteria of mesenchymopathic type of CIP is poorly defined with reference to the mean number of ICCs and distribution of the ICCs. It is also not clear whether the multifocal decrease of ICCs in our patient is the primary cause of CIP or secondary to continuous colon dilation.

In summary, we have reported a case of CIP which was aggravated severely by pregnancy. However, in such cases, radiologic studies and administration of neostigmine may be limited due to the associated fetal risks. Endoscopic decompression can be performed repetitively with minimal fetal risks until elective surgery can be performed.

\section{Conflicts of Interest}

The authors have no financial conflicts of interest.

\section{REFERENCES}

1. Faulk DL, Anuras S, Christensen J. Chronic intestinal pseudoobstruction. Gastroenterology 1978;74(5 Pt 1):922-931.

2. Luckas M, Buckett W. Acute colonic pseudo-obstruction in the obstetric patient. Br J Hosp Med 1997;57:378-381.

3. ACOG Committee on Obstetric Practice. ACOG Committee Opinion. Number 299, September 2004 (replaces No. 158, September 1995). Gu idelines for diagnostic imaging during pregnancy. Obstet Gynecol 2004; 104:647-651.

4. Ponec RJ, Saunders MD, Kimmey MB. Neostigmine for the treatment of acute colonic pseudo-obstruction. N Engl J Med 1999;341:137-141.

5. Lyford G, Foxx-Orenstein A. Chronic intestinal pseudoobstruction. Curr Treat Options Gastroenterol 2004;7:317-325.

6. Geller A, Petersen BT, Gostout CJ. Endoscopic decompression for acu te colonic pseudo-obstruction. Gastrointest Endosc 1996;44:144-150.

7. Classen M, Tytgat GN, Lightdale CL, et al. Gastroenterological Endoscopy. 2nd ed. New York: Thieme; 2010.

8. Cappell MS. Risks versus benefits of gastrointestinal endoscopy during pregnancy. Nat Rev Gastroenterol Hepatol 2011;8:610-634.

9. De Giorgio R, Sarnelli G, Corinaldesi R, et al. Advances in our underst anding of the pathology of chronic intestinal pseudo-obstruction. Gut 2004;53:1549-1552.

10. Choe EK, Park SH, Park KJ. Colonic pseudo-obstruction with distinct transitional zone in adult constipation patients: pathological analysis and results of surgical treatment. Am Surg 2011;77:736-742.

11. Do MY, Myung SJ, Park HJ, et al. Novel classification and pathogenetic analysis of hypoganglionosis and adult-onset Hirschsprung's disease. Dig Dis Sci 2011;56:1818-1827. 